\title{
Resveratrol ameliorates bisphenol A-induced testicular toxicity in adult male rats: A stereological and functional study
}

Hossein Bordbar

Shiraz University of Medical Sciences

Seyedeh-Saeedeh Yahyavi

Shiraz University of Medical Sciences

Ali Noorafshan

Shiraz University of Medical Sciences

Elham Aliabadi

Shiraz University of Medical Sciences

Maryam Naseh ( $\nabla$ naseh@sums.ac.ir)

Shiraz University of Medical Sciences https://orcid.org/0000-0003-4254-5175

\section{Research Article}

Keywords: Bisphenol A, resveratrol, testicular toxicity, sperm parameters, stereology

Posted Date: March 2nd, 2022

DOI: https://doi.org/10.21203/rs.3.rs-1398067/v1

License: (c) (i) This work is licensed under a Creative Commons Attribution 4.0 International License. Read Full License 


\section{Abstract}

Bisphenol A (BPA) is one of the most widely used synthetic chemicals worldwide. BPA as an endocrine disruptor affects the reproductive systems through estrogenic and antiandrogenic proprieties. Resveratrol (RES) as a natural polyphenol and potent antioxidant exhibits the protective effects against reproductive toxicity by inhibition of oxidative stress. 48 male rats divided into eight groups $(n=6)$, including control (CON), Olive oil ( $0.5 \mathrm{ml} /$ day), Carboxy methylcellulose (CMC) $(1 \mathrm{ml}$ of $10 \mathrm{~g} / \mathrm{l}), \mathrm{RES}(100 \mathrm{mg} / \mathrm{kg} / \mathrm{day})$, low dose of BPA ( $25 \mathrm{mg} / \mathrm{kg} /$ day), high dose of BPA (50 mg/kg/day), low dose of BPA + RES, and high dose of $\mathrm{BPA}+\mathrm{RES}$. All treatment were done orally by gavages per day for 56 days. At the end of the 8th week, blood samples were collected for hormone assays. Then, the sperm parameters were analyzed, and the left testis was removed for stereological study. We showed a significant decrease in sperm parameters in the low and high doses of BPA groups compared to control group $(P<0.05)$. The volume of testicular components as well as the diameter and length of seminiferous tubules significantly reduced (11-64\%), and the total number of the testicular cell types decreased (34-67\%) on average in the low and high doses of BPA groups. Moreover, serum FSH, LH, and testosterone hormones concentration showed a significant reduction in both doses of BPA groups $(P<0.01)$. Nonetheless, treatment with RES could ameliorate all the above-mentioned changes in the low and high doses of BPA groups $(P<0.05)$. RES could prevent BPA-induced testicular structural changes and sperm quality via improving gonadotropin hormones and testosterone levels.

\section{1- Introduction}

Bisphenol A (BPA) is one of the most widely used synthetic chemicals worldwide. It is found in large amount of consumer products such as polycarbonate plastics, food packaging, epoxy resins, linings of cans, dental sealants and thermal paper (Kang et al. 2003; Vandenberg et al. 2007; Biedermann et al. 2010). Public health has raised concerns about the widespread applications and toxic effects of BPA (Konieczna et al. 2015). Exposure of BPA can occur directly or indirectly through inhalation, dermal exposure and ingestion (Kang et al. 2006; Williams et al. 2014). It has been reported that the main rout of exposure in humans is oral, which accounts about $90 \%$ of BPA exposures. It has been shown that BPA contributes to the cause of several endocrine disorders including reproductive dysfunction, infertility, precocious puberty and hormone dependent tumors (Matuszczak et al. 2019; Santiago et al. 2021). Evidences suggest that BPA exerts the toxic effects on the reproductive system via different mechanisms. BPA as an endocrine disruptor seems to mediate reproductive failure through estrogenic and antiandrogenic proprieties (Maffini et al. 2006). BPA can interfere with estrogenic signaling pathways by interacting with estrogen receptors (ERs), or by producing a small but potent estrogenic metabolite (Alonso-Magdalena et al. 2012). BPA can also bind to the androgen receptor (AR) as an antagonist (Lee et al. 2003), which can disrupt the hypothalamic-pituitary-testicular axis, thereby affecting gene expression and the enzymatic activity of testicular steroidogenesis, leading to hypogonadotropic hypogonadism (Chimento et al. 2014; Wisniewski et al. 2015). 
In this regard, several animal studies have also confirmed the reproductive toxicity of BPA in rats and mice (Takahashi and Oishi 2001; Tohei et al. 2001; El Ghazzawy et al. 2011). It has been demonstrated that BPA decreases testis weight, reduces diameter and thickness of seminiferous tubules and leads to compromised spermatogenesis. These morphological alterations and abnormal spermatogenesis seem to be induced by the reduction of reproductive hormone production and promotion of germ cell apoptosis (Akingbemi et al. 2004; Urriola-Muñoz et al. 2014). On the other hand, exposure to BPA is related to the reduced activity of antioxidant enzymes, which could contribute to oxidative stress and sperm damage (Hulak et al. 2013; Meli et al. 2020).

Resveratrol (RES; trans-3,5,4'-trihidroxy-trans-stilbene), as a natural polyphenol and potent antioxidant is found in a wide range of foods, especially grapes, berries, and peanuts (Burns et al. 2002). Several reports have demonstrated that RES exhibits the protective effects against reproductive toxicity by suppressing lipid peroxidation (Collodel et al. 2011; de Oliveira et al. 2019). Moreover, RES may improve sperm count and motility, as well as decrease germ cell apoptosis by stimulating the hypothalamic-pituitary-gonad axis and enhancing blood testosterone levels (Shati 2019). Accordingly, this study was designed to evaluate the protective effects of RES against destructive effects of low (25 mg/kg/day) and high doses (50 mg/kg/day) of BPA on the structure and function of testis using stereological assessment, hormonal measurements, and quantitative-qualitative study of sperm parameters.

\section{2- Materials And Methods \\ 2.1. Animals}

Forty eight male Sprague-Dawley rats (180-210 g) were purchased from the Animal Laboratory Center of Shiraz University of Medical Sciences (SUMS). The animals were kept under standard conditions at room temperature $\left(22 \pm 2^{\circ} \mathrm{C}\right)$, with normal humidity and $12-12 \mathrm{~h}$ light-dark cycles. They also had free access to standard food and water. The animal procedures were performed under the standard rules established by the Animal Care and Ethics Committee of SUMS (IR.SUMS.REC.1398.392).

\subsection{Experimental design}

The rats were randomly divided into eight groups $(n=6)$; Group I: received distilled water as control (CON), Group II: Olive oil ( $0.5 \mathrm{ml} /$ day), Group III: Carboxy methylcellulose (CMC) (1 ml of $10 \mathrm{~g} / \mathrm{l})$, Group IV: RES ( $100 \mathrm{mg} / \mathrm{kg} /$ day) suspended in CMC, Group V: low dose of BPA ( $25 \mathrm{mg} / \mathrm{kg} /$ day) dissolved in olive oil, Group Vl: high dose of BPA ( $50 \mathrm{mg} / \mathrm{kg} /$ day) dissolved in olive oil, Group VII: low dose of BPA plus RES ( $100 \mathrm{mg} / \mathrm{kg} /$ day), and Group VIII: high dose of BPA plus RES $(100 \mathrm{mg} / \mathrm{kg} /$ day). It should be noted that the animals in all groups were treated orally by gavages per day for 56 days.

\subsection{Hormone measurements}

At the end of the 8th week, Blood samples were collected and stored in heparin-free tubes. Then, the samples were centrifuged at $3500 \mathrm{rpm}$ for $15 \mathrm{~min}$. The serum was obtained and stored at $-70^{\circ} \mathrm{C}$ for 
subsequent hormone evaluation. The serum levels of $\mathrm{FSH}, \mathrm{LH}$, and testosterone concentrations were determined by rat ELISA kits using a micro plate reader (Biotek, USA).

\subsection{Spermatozoa counts, morphology and motility}

The rats' ductus deferens were evaluated for semen analysis as previously described (Aminsharifi et al. 2016).

\subsection{Stereological study}

On day 56, the left testis was removed and weighed. Its primary volume "V (testicle)" was measured by the immersion method (Mandarim-de-Lacerda 2003). Then, the samples were fixed in $4 \%$ buffered formaldehyde solution for stereological studies. The orientator method was applied to obtain Isotropic Uniform Random (IUR) sections (Mandarim-de-Lacerda 2003). About 8-12 slabs in each testis were collected through this procedure. A circle was punched out from a random testis slab by a trocar for estimation of the tissue shrinkage. After tissue processing and paraffin embedding, 5 and $25 \mu \mathrm{m}$ sections were cut by the microtome and were stained using Hematoxylin-Eosin. The areas of the circles were measured before processing (unshrunken) and after processing (shrunken) and finally, the degree of shrinkage "d (shr)" was calculated by the following formula:

$\mathrm{d}($ shr $)=1-[\text { Area(after) } / \text { Area(before) }]^{1.5}$

Then, the total volume of the testis was evaluated with regard to tissue shrinkage [V(shrunken)] using the following formula:

$\mathrm{V}($ shrunken $)=\mathrm{V}($ unshrunken $) \times[1-\mathrm{d}($ shr $)]$

\subsubsection{Estimation of the testicular components volume}

The volume density of the testis sections was analyzed by a video microscopy system. In doing so, the point grid was superimposed on the microscopic images of the sections ( $5 \mu \mathrm{m}$ thickness) on a monitor by the software designed at the Histomorphometry and Stereology Research Center. The volume density "Vv (structure/testis)" of the testicular components, including seminiferous tubules, interstitial tissue, and germinal epithelium, was estimated by the point counting method (Tschanz et al. 2014). Finally, the total volume of each component was obtained by the following formula:

$\mathrm{V}($ structure $)=\mathrm{V} v($ structure/testis $) \times \mathrm{V}($ shrunken $)$

\subsubsection{Estimation of the length and diameter of seminiferous tubules}

The length density (Lv) of the seminiferous tubules was measured on the sampled tubules in an unbiased counting frame applied on the $5 \mu \mathrm{m}$ thick sections (Dalgaard et al. 2002), and calculated by the following formula: 
$L v=2 \Sigma Q /[\Sigma P \times(a / f)]$

Where " $\Sigma Q$ " is the total number of the selected tubules, " $\Sigma \mathrm{P}$ " represents the total points superimposed on the testis, and " $a / f$ " indicates the area of the counting frame. The total length of the seminiferous tubules "L(tubules)" was calculated by multiplying the lengths density (Lv) by V(structure) (Howard and Reed 2004).

$\mathrm{L}($ tubules $)=\mathrm{Lv} \times \mathrm{V}($ structure $)$

The diameter of the seminiferous tubules was also measured on the sampled tubules in the counting frame. The diameter was measured perpendicularly to the long axis of the tubules where the tubules were widest (Dalgaard et al. 2002). An average of 100 tubules were counted per testis.

\subsubsection{Estimation of number of testicular cell types}

A computer linked to a light microscope (Nikon E200, Japan) with 40x oil lens (NA $=1.4)$ was used to assess the total number of testicular cell types, including spermatogonia ( $A$ and $B$ ), spermatocytes, spermatids (round and long), sertoli and leydig cells. The total number of the testicular cell types was calculated using the optical disector method applied on the $25 \mu \mathrm{m}$ thick sections (Wreford 1995). In so doing, the microscopic fields were scanned by moving the microscope stage at equal distances in $\mathrm{X}$ and $Y$ directions based on systematic uniform random sampling. The movement in $Z$ direction was also performed using a microcator (MT12, Heidenhain, Germany) fixed on the microscope stage. The Z-axis distribution from the sampled cells in different focal planes was plotted to determine the guard zones and disector's height (von Bartheld 2012). The numerical density (Nv) was estimated using the following formula:

$N v=\Sigma Q /(\Sigma A \times h) \times(t / B A)$

Where " $\Sigma Q$ " was the number of each cell type nuclei coming into focus, " $\Sigma A$ " indicated the total area of the unbiased counting frame, " $h$ " represented the disector's height, " $t$ " was the mean section thickness, and "BA" was the microtome block advance. Finally, the total number of the testicular cell types was calculated by multiplying the numerical density $(\mathrm{Nv})$ by $\mathrm{V}$ (structure):

$\mathrm{N}($ cells $)=\mathrm{Nv} \times \mathrm{V}($ structure $)$

Where, $\mathrm{V}$ (structure) was the total volume of the germinal epithelium for the germinal layer cells and the total volume of the interstitial tissue for the leydig cells.

\subsection{Statistical analysis}

The results were analyzed by one-way analysis of variance (ANOVA) and Tukey's post hoc test using Graph Pad Prism 6 software (San Diego, CA, USA). P $<0.05$ was considered to be statistically significant.

\section{3- Results}




\subsection{Spermatozoa count, normal morphology and motility}

According to Table 1, a significant decrease was observed in the count, percentage of normal morphology, and motility of spermatozoa in the rats exposed to low and high doses of BPA groups compared to control group $(P<0.05$ and $P<0.01$, respectively). However, these parameters in the low dose of BPA + RES and high dose of BPA + RES groups improved compared to the BPA groups $(P<0.01$ and $P<0.05$, respectively).

Table 1

Mean \pm SEM of the Count (×106), Normal morphology (\%), Motility (\%), and Immotile (\%) in the experimental groups $(n=6)$.

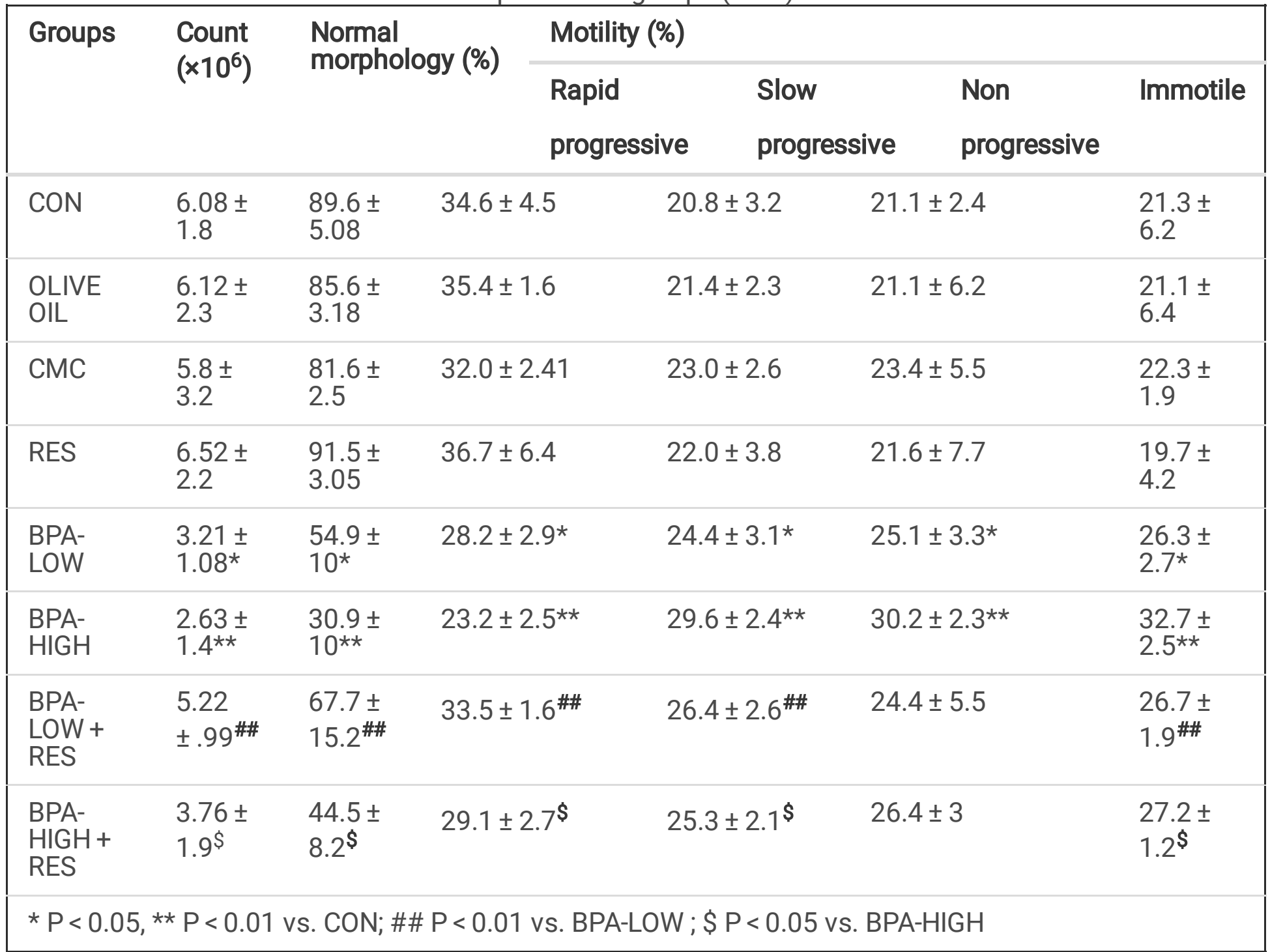

\subsection{Stereological assays \\ 3.2.1. The volume of the testicle}


The results showed a significant reduction in the testicle volume by $11.7 \%$ and $13.5 \%$ in the rats exposed to low and high doses of BPA compared to the control groups, respectively $(P<0.01$ and $P<0.001)$. However, the testis volume recovered considerably in the animals that received low dose of BPA + RES group compared to low dose of BPA group $(P<0.01)$ (Fig. 1A).

\subsubsection{The volume of germinal epithelium}

The total epithelial volume in rats treated with low and high doses of BPA decreased $43 \%$ and $64 \%$ in comparison to the control group, respectively $(P<0.001)$. Treatment with RES ameliorated the epithelial volume changes in the low or high doses of BPA groups $(P<0.001)$ (Fig. 1B).

\subsubsection{The volume of interstitial tissue}

The results indicated that the interstitial tissue volume reduced $25.3 \%$ and $27.3 \%$ in the low and high doses of compared to the control groups, respectively $(P<0.01$ and $P<0.05)$. However, this parameter significantly was increased in the rats treated with RES in the low or high doses of BPA groups $(P<0.05)$ (Fig. 1C).

\subsubsection{The volume of seminiferous tubules}

A significant reduction was seen in the total volume of seminiferous tubules by $26.2 \%$ and $34 \%$ in the low and high doses of BPA compared to the control groups, respectively $(P<0.01$ and $P<0.001)$.

Nevertheless, seminiferous volume significantly was ameliorated in the low dose of BPA + RES and high dose of BPA + RES groups compared to the BPA groups ( $P<0.01$ and $P<0.05$, respectively) (Fig. 1D).

\subsubsection{Diameter of the seminiferous tubules}

The diameter of the seminiferous tubules decreased $29.7 \%$ and $37.3 \%$ in rats treated with low and high doses of BPA compared to the control group $(P<0.001)$. Treatment with RES increased this parameter in the low and high doses of BPA groups $(P<0.01)$ (Fig. 2A).

\subsubsection{Length of the seminiferous tubules}

The results showed that length of the seminiferous tubules have reduced $20.6 \%$ and $29.8 \%$ in the low and high doses of BPA compared to the control groups ( $P<0.01$ and $P<0.001$, respectively). Nonetheless, the tubules length significantly was improved in the rats treated with RES in the low and high doses of BPA groups ( $\mathrm{P}<0.05$ and $\mathrm{P}<0.01$, respectively) (Fig. 2B).

\subsubsection{Number of Spermatogonia $A$ and $B$}

The total number of spermatogonia A reduced by $40.03 \%$ and $55.2 \%$, and spermatogonia B by $51.27 \%$ and $70.05 \%$ in the low and high doses of BPA compared to the control groups, respectively $(P<0.001)$. However, treatment with RES increased these cells in the low and high doses of BPA groups $(P<0.001)$ (Fig. 3A and B).

\subsubsection{Number of Spermatocytes}


Statistical analysis showed $34.85 \%$ and $53 \%$ reduction in the number of spermatocytes for both the low and high doses of BPA compared to the control groups $(P<0.001)$. Treatment with RES ameliorated these changes in the low dose of BPA + RES and high dose of BPA + RES groups compared to the BPA groups $(P<0.01$ and $P<0.001$, respectively) (Fig. $3 C$ ).

\subsubsection{Number of Round and Long Spermatid}

The number of round spermatids decreased by $40.76 \%$ and $66.72 \%$, and long spermatids by $28.7 \%$ and $60.35 \%$ in the low and high doses of BPA, respectively compared to the control groups $(P<0.001)$.

Moreover, ameliorative effects of RES on the number of these cells were seen in rats treated with low and high doses of BPA groups $(P<0.001)$ (Fig. 3D and E).

\subsubsection{Number of Leyding and Sertoli cells}

A significant reduction was seen in the number of Leydig cells by $45.78 \%$ and $62.85 \%$, and Sertoli cells by $32.28 \%$ and $52.76 \%$ in the low and high doses of BPA than those of the control groups, respectively $(\mathrm{P}<$ 0.001). Treatment with RES recovered the number of Leydig cells and Sertoli cells in the low dose of BPA $+\operatorname{RES}(P<0.01$ and $P<0.05$, respectively), and high dose of BPA + RES $(P<0.001$ and $P<0.01$, respectively) groups compared to the BPA groups (Fig. 3F and $\mathrm{G}$ ).

\subsection{Qualitative changes}

Qualitative evaluation of the testis has been presented in Figure. 4. The histological sections of the low and high doses of BPA rats showed the structural changes, including atrophy and reduced number of seminiferous tubules. Concomitant treatment of the these groups with RES ameliorated these destructive effects.

\subsection{Hormone assays}

The gonadotropins assessment showed a significant reduction in serum $\mathrm{LH}$ and $\mathrm{FSH}$ levels in the low dose of BPA $(P<0.001$ and $P<0.01$, respectively), and high dose of BPA $(P<0.001)$ groups compared to the control group. Also, the testosterone concentration of the rats given low or high doses of BPA were lower than in the control group $(\mathrm{P}<0.001)$. The RES exposure led to significant increase in the serum $\mathrm{LH}$ and testosterone levels in the low dose of BPA $(P<0.01$ and $P<0.001$, respectively), and high dose of BPA $(P<0.05$ and $P<0.001$, respectively) groups, while the serum FSH levels significantly increased only in the high dose of BPA + RES group $(P<0.05)$ (Fig. 5).

\section{4- Discussion}

The current study revealed the ameliorative effects of RES on testicular damage induced by BPA in rats. The first part of our findings showed the deleterious effects of two doses of BPA, 25 and $50 \mathrm{mg} / \mathrm{kg} / \mathrm{day}$ for 8 weeks, on sperm quality and structural changes of the testis. The earlier studies showed that 50 $\mathrm{mg} / \mathrm{kg} /$ day is considered as maximum permissible dose that have no observable side effect on 
reproductive and developmental toxicity (FAO/WHO 2010). But we found that ingestion of BPA at these dosages had adverse effects on count, morphology, and motility of spermatozoa. In line with our results, it has been observed a reduction in epididymal sperm motility and count in the rats exposed to BPA at the 10 and $50 \mathrm{mg} / \mathrm{kg}$ in a dose dependent manner (Kourouma et al. 2014). Also, it has been demonstrated that BPA at 5 and $25 \mathrm{mg} / \mathrm{kg} /$ day reduced sperm production, reserves and transit time through the epididymis (Wisniewski et al. 2015). Moreover, long-term exposure to $0.2 \mathrm{mg} / \mathrm{kg}$ BPA in rats led to decreased sperm count and inhibited spermiation (Liu et al. 2013).

The reduction in sperm count and quality are in accord with decreased stereological parameters. The changes of structural indices including volume, diameter and length of seminiferous tubules suggest the atrophy of these tubules and testicular abnormalities due to BPA. Loss of the germinal epithelial cells was also seen after exposure to both doses of BPA. A reduction in germinal epithelial volume could be a consequence of decline in the number of germinal cells. The reduction in sperm production could be related to the disruption of spermatogenesis. Jin et al., 2013 also reported that BPA exposure could decrease sperm count via the reduction in type A spermatogonial, spermatocytes and spermatids. BPA impaired spermatogenesis through suppressing reproductive hormones and activating germ cells apoptosis mediated by Fas/FasL signaling pathway (Jin et al. 2013; Wang et al. 2014). Sertoli cells are another type of cells in the seminiferous tubules, which have a supportive and nutrient function. Since, Sertoli cells can affect the proliferation and differentiation of germinal cells, and also help in the process of spermatogenesis. So, it seems the loss of these supporting cells could be led to deficiency of supportive functions in BPA-treated rat, and cause the loss of spermatogenic cells. It has been indicated that Sertoli cells are targets of pituitary-derived follicle-stimulating hormone (FSH) and testosterone to transduce signals into paracrine regulation of spermatogenesis (Walker and Cheng 2005; Smith and Walker 2014; Oduwole et al. 2018). Accordingly, Sertoli cell depletion following BPA treatment in present study may be due to a decrease in FSH and testosterone levels. On the other hand, testosterone secretion is produced in Leydig cells of testicular interstitium in response to luteinizing hormone (LH) ( $\mathrm{Li}$ et al. 2021). Therefore, the lack of LH stimulation in BPA-treated groups could justify the reduction of Leydig cells and interstitial tissue atrophy and also the decrease of testosterone production. Testosterone is an essential hormone to maintain normal spermatogenesis and prevention of germ cell apoptosis in adult rats (Aitken and Roman 2009). So, it is reasonable to assume that the inhibition of reproductive hormones production may have contributed to spermatogenesis impairment induced by BPA. Similarly, BPA could cause defective spermatozoa by disruption of the hypothalamic-pituitary-gonadal axis, causing a state of hypogonadotropic hypogonadism (Jin et al. 2013; Mohamed and Arafa 2013; Wisniewski et al. 2015). Another possible hypothesis may be involved in spermatogenesis dysfunction is the effects of BPA-induced oxidative damage. BPA exposure could induce ROS production by reducing the activity of the antioxidant system (Chitra et al. 2003). The adverse effects of BPA on sperm count and quality due to oxidative stress have been described by previous studies (Mohamed and Arafa 2013; Kourouma et al. 2014).

The second step of our study demonstrated the protective effects of RES against BPA-induced testicular structural changes and sperm quality. Our results indicated that the concomitant treatment of the BPA 
groups with RES for 8 weeks could significantly restore the sperm parameters and prevent testicular atrophy and apoptosis of the testicular cell types. Furthermore, RES enhanced testosterone, FSH, and LH levels in BPA groups. The improvement of testicular structural and sperm quality seem to be related to increased gonadotropin hormones and testosterone levels. Consistent with these findings, previous reports have also shown that levels of FSH, $\mathrm{LH}$, and testosterone increase in RES-treated rats, thereby improving sperm parameters and testicular apoptosis (Shati 2019). Also, another earlier study claimed that RES could ameliorate negative effects against BPA-induced reproductive toxicity in mice via reducing oxidative stress (Golmohammadi et al. 2021). Our results support the contribution of reproductive hormones in the ameliorative effects of RES on BPA-induced testicular toxicity in rats. Meanwhile, a reduction of oxidative stress, as shown by other studies, may also be involved in RES protective effects, which required further studies to be confirmed.

\section{5- Conclusion}

In conclusion, the present study demonstrated the protective effects of RES against BPA-induced testicular structural changes and sperm quality via improving gonadotropin hormones and testosterone levels.

\section{Declarations}

\section{ACKNOWLEDGMENTS}

This article was extracted from the thesis of Seyedeh-Saeedeh Yahyavi's, M.Sc. in Anatomy. This work was performed at the Histomorphometry and Stereology Research Center and was financially supported by grant No. 97-01-21-18338 from Shiraz University of Medical Sciences. Hereby, the authors would like to thank Ms. A. Keivanshekouh at the Research Consultation Center (RCC) of Shiraz University of Medical Sciences for improving the use of English in the manuscript.

\section{CONFLICT OF INTEREST}

The authors have no conflict of interest to report.

\section{ETHICAL STATEMENTS}

All experimental procedures in the current study were done in accordance with the National Institutes of Health guide for the care and use of laboratory animals (NIH Publications No. 8023, revised 1978) and were approved by the Medical and Research Ethics Committee of Shiraz University of Medical Sciences, Shiraz, Iran (Approval No. IR.SUMS.REC.1398.392).

\section{INFORMED CONSENT}

Written informed consent was obtained from all study participants. 


\section{References}

1. Aitken RJ, Roman SD (2009) Antioxidant systems and oxidative stress in the testes. Molecular mechanisms in spermatogenesis:154-171

2. Akingbemi BT, Sottas CM, Koulova Al, et al. (2004) Inhibition of testicular steroidogenesis by the xenoestrogen bisphenol $\mathrm{A}$ is associated with reduced pituitary luteinizing hormone secretion and decreased steroidogenic enzyme gene expression in rat Leydig cells. Endocrinology 145(2):592-603

3. Alonso-Magdalena P, Ropero AB, Soriano S, et al. (2012) Bisphenol-A acts as a potent estrogen via non-classical estrogen triggered pathways. Molecular and cellular endocrinology 355(2):201-207

4. Aminsharifi A, Hekmati P, Noorafshan A, et al. (2016) Scrotal cooling to protect against cisplatininduced spermatogenesis toxicity: preliminary outcome of an experimental controlled trial. Urology 91:90-98

5. Biedermann S, Tschudin P, Grob K (2010) Transfer of bisphenol A from thermal printer paper to the skin. Analytical and bioanalytical chemistry 398(1):571-576

6. Burns J, Yokota T, Ashihara $\mathrm{H}$, et al. (2002) Plant foods and herbal sources of resveratrol. Journal of agricultural and food chemistry 50(11):3337-3340

7. Chimento A, Sirianni R, Casaburi I, Pezzi V (2014) Role of estrogen receptors and G protein-coupled estrogen receptor in regulation of hypothalamus-pituitary-testis axis and spermatogenesis. Frontiers in endocrinology 5:1

8. Chitra K, Latchoumycandane C, Mathur P (2003) Induction of oxidative stress by bisphenol A in the epididymal sperm of rats. Toxicology 185(1-2):119-127

9. Collodel G, Federico M, Geminiani M, et al. (2011) Effect of trans-resveratrol on induced oxidative stress in human sperm and in rat germinal cells. Reproductive Toxicology 31(2):239-246

10. Dalgaard M, Pilegaard K, Ladefoged $O$ (2002) In Utero Exposure to Diethylstilboestrol or 4-nNonylphenol in Rats: Number of Sertoli Cells, Diameter and Length of Seminiferous Tubules Estimated by Stereological Methods. Pharmacology \& toxicology 90(2):59-65

11. de Oliveira FA, Costa WS, Sampaio FJ, Gregorio BM (2019) Resveratrol attenuates metabolic, sperm, and testicular changes in adult Wistar rats fed a diet rich in lipids and simple carbohydrates. Asian Journal of Andrology 21(2):201

12. El Ghazzawy IF, Meleis AE, Farghaly EF, Solaiman A (2011) Histological study of the possible protective effect of pomegranate juice on bisphenol-A induced changes of the caput epididymal epithelium and sperms of adult albino rats. Alexandria Journal of Medicine 47(2):125-137

13. FAO/WHO (2010) Reproductive and Developmental Toxicity of Bisphenol A in Mammalian Species. WHO Press Ottawa

14. Golmohammadi MG, Khoshdel F, Salimnejad R (2021) Protective effect of resveratrol against bisphenol A-induced reproductive toxicity in male mice. Toxin Reviews:1-9

15. Howard V, Reed M (2004) Unbiased stereology: three-dimensional measurement in microscopy. Garland Science 
16. Hulak M, Gazo I, Shaliutina A, Linhartova P (2013) In vitro effects of bisphenol A on the quality parameters, oxidative stress, DNA integrity and adenosine triphosphate content in sterlet (Acipenser ruthenus) spermatozoa. Comparative Biochemistry and Physiology Part C: Toxicology \& Pharmacology 158(2):64-71

17. Jin P, Wang X, Chang F, et al. (2013) Low dose bisphenol A impairs spermatogenesis by suppressing reproductive hormone production and promoting germ cell apoptosis in adult rats. Journal of biomedical research 27(2):135

18. Kang J-H, Kito K, Kondo F (2003) Factors influencing the migration of bisphenol A from cans. Journal of food protection 66(8):1444-1447

19. Kang J-H, Kondo F, Katayama Y (2006) Human exposure to bisphenol A. Toxicology 226(2-3):79-89

20. Konieczna A, Rutkowska A, Rachon D (2015) Health risk of exposure to Bisphenol A (BPA). Roczniki Państwowego Zakładu Higieny 66(1)

21. Kourouma A, Peng D, Chao Q, et al. (2014) Bisphenol A induced reactive oxygen species (ROS) in the liver and affect epididymal semen quality in adults Sprague-Dawley rats. Journal of Toxicology and Environmental Health Sciences 6(4):103-112

22. Lee HJ, Chattopadhyay S, Gong E-Y, et al. (2003) Antiandrogenic effects of bisphenol A and nonylphenol on the function of androgen receptor. Toxicological Sciences 75(1):40-46

23. Li X, Zhu Q, Wen Z, et al. (2021) Androgen and Luteinizing Hormone Stimulate the Function of Rat Immature Leydig Cells Through Different Transcription Signals. Frontiers in endocrinology 12:205

24. Liu C, Duan W, Li R, et al. (2013) Exposure to bisphenol A disrupts meiotic progression during spermatogenesis in adult rats through estrogen-like activity. Cell death \& disease 4(6):e676-e676

25. Maffini MV, Rubin BS, Sonnenschein C, Soto AM (2006) Endocrine disruptors and reproductive health: the case of bisphenol-A. Molecular and cellular endocrinology 254:179-186

26. Mandarim-de-Lacerda CA (2003) Stereological tools in biomedical research. Anais da Academia brasileira de Ciências 75(4):469-486

27. Matuszczak E, Komarowska MD, Debek W, Hermanowicz A (2019) The impact of bisphenol A on fertility, reproductive system, and development: a review of the literature. International journal of endocrinology 2019

28. Meli R, Monnolo A, Annunziata C, et al. (2020) Oxidative stress and BPA toxicity: An antioxidant approach for male and female reproductive dysfunction. Antioxidants 9(5):405

29. Mohamed DA, Arafa MH (2013) Testicular toxic changes induced by bisphenol A in adult albino rats: a histological, biochemical, and immunohistochemical study. Egyptian Journal of Histology 36(1):233-245

30. Oduwole 0O, Peltoketo H, Huhtaniemi IT (2018) Role of follicle-stimulating hormone in spermatogenesis. Frontiers in endocrinology 9:763

31. Santiago J, Silva JV, Santos MA, Fardilha M (2021) Fighting Bisphenol A-Induced Male Infertility: The Power of Antioxidants. Antioxidants 10(2):289 
32. Shati AA (2019) Resveratrol improves sperm parameter and testicular apoptosis in cisplatin-treated rats: effects on ERK1/2, JNK, and Akt pathways. Systems biology in reproductive medicine 65(3):236-249

33. Smith LB, Walker WH The regulation of spermatogenesis by androgens. In: Seminars in cell \& developmental biology, 2014. vol 30. Elsevier, p 2-13

34. Takahashi O, Oishi S (2001) Testicular toxicity of dietary 2, 2-bis (4-hydroxyphenyl) propane (bisphenol A) in F344 rats. Archives of toxicology 75(1):42-51

35. Tohei A, Suda S, Taya K, et al. (2001) Bisphenol A inhibits testicular functions and increases luteinizing hormone secretion in adult male rats. Experimental biology and medicine 226(3):216-221

36. Tschanz S, Schneider JP, Knudsen L (2014) Design-based stereology: planning, volumetry and sampling are crucial steps for a successful study. Annals of Anatomy-Anatomischer Anzeiger 196(1):3-11

37. Urriola-Muñoz P, Lagos-Cabré R, Moreno RD (2014) A mechanism of male germ cell apoptosis induced by bisphenol-A and nonylphenol involving ADAM17 and p38 MAPK activation. PLoS One 9(12): 113793

38. Vandenberg LN, Hauser R, Marcus M, et al. (2007) Human exposure to bisphenol A (BPA). Reproductive toxicology 24(2):139-177

39. von Bartheld CS (2012) Distribution of particles in the z-axis of tissue sections: relevance for counting methods. NeuroQuantology: an interdisciplinary journal of neuroscience and quantum physics 10(1):66

40. Walker WH, Cheng J (2005) FSH and testosterone signaling in Sertoli cells. Reproduction 130(1):1528

41. Wang P, Luo C, Li Q, et al. (2014) Mitochondrion-mediated apoptosis is involved in reproductive damage caused by BPA in male rats. Environmental toxicology and pharmacology 38(3):1025-1033

42. Williams C, Bondesson M, Krementsov DN, Teuscher C (2014) Gestational bisphenol A exposure and testis development. Endocrine disruptors 2(1):e29088

43. Wisniewski P, Romano RM, Kizys MM, et al. (2015) Adult exposure to bisphenol A (BPA) in Wistar rats reduces sperm quality with disruption of the hypothalamic-pituitary-testicular axis. Toxicology 329:1-9

44. Wreford NG (1995) Theory and practice of stereological techniques applied to the estimation of cell number and nuclear volume in the testis. Microscopy research and technique 32(5):423-436

\section{Figures}

\section{Figure 1}


The box plots represents the volume of the testis (A), germinal epithelium (B), interstitial tissue (C), and seminiferous tubules (D) in the control (CON), olive oil, carboxy methylcellulose (CMC), resveratrol (RES), low dose of Bisphenol A (BPA), high dose of BPA, low dose of BPA+RES, and high dose of BPA+RES groups. $n=6$ in each group. Data are presented as mean $\pm S E$. ${ }^{*} p<0.05, * * p<0.01$, and ${ }^{* \star *} p<0.001$ vs. control; $\# p<0.05, \# \# p<0.01$, and \#\#\#p $<0.001$ vs. low dose of BPA; $\$ p<0.05$ and $\$ \$ \$ p<0.001$ vs. high dose of BPA.

A

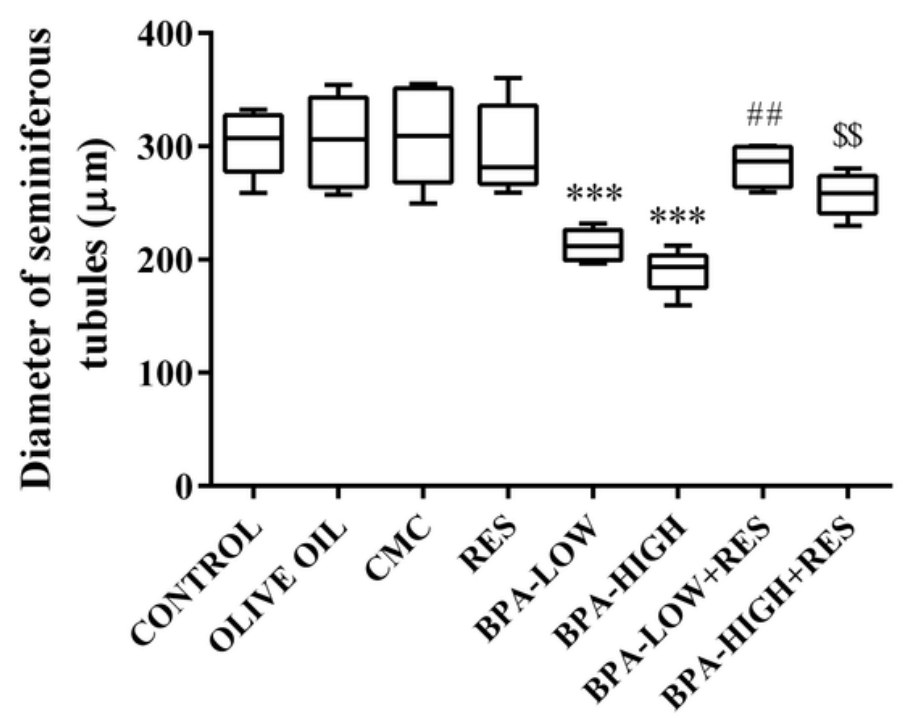

B

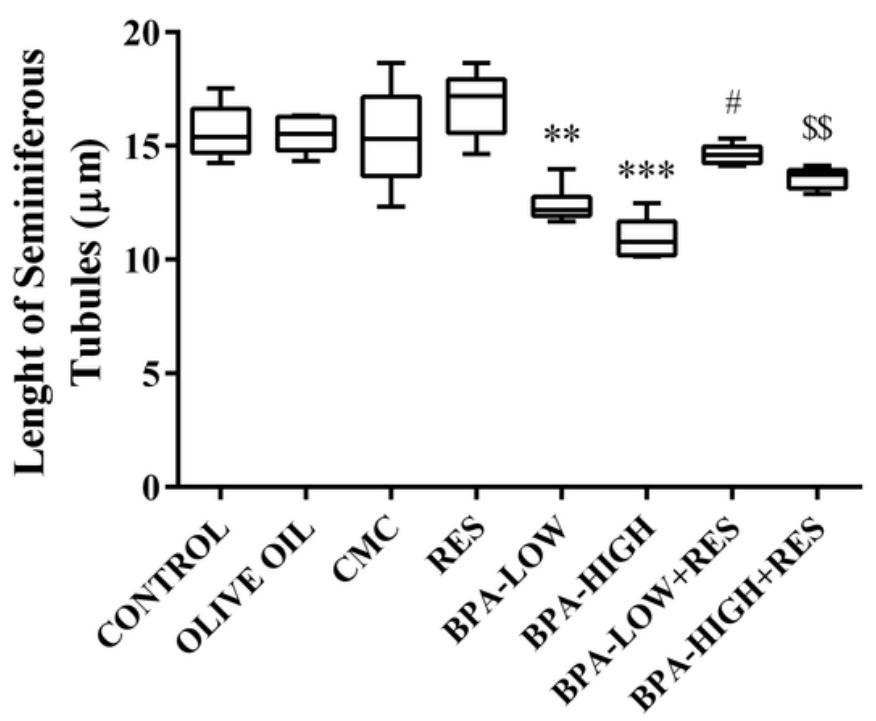

Figure 2

The box plots shows the diameter (A), and the length (B) of seminiferous tubules in the control (CON), olive oil, carboxy methylcellulose (CMC), resveratrol (RES), low dose of Bisphenol A (BPA), high dose of BPA, low dose of BPA+RES, and high dose of BPA+RES groups. $n=6$ in each group. Data are presented as mean \pm SE. ${ }^{\star \star} p<0.01$, and ${ }^{\star \star \star} p<0.001$ vs. control; $\# p<0.05$, \#\#p $<0.01 v$ s. low dose of BPA; $\$ \$ p<$ 0.01 vs. high dose of BPA. 
A

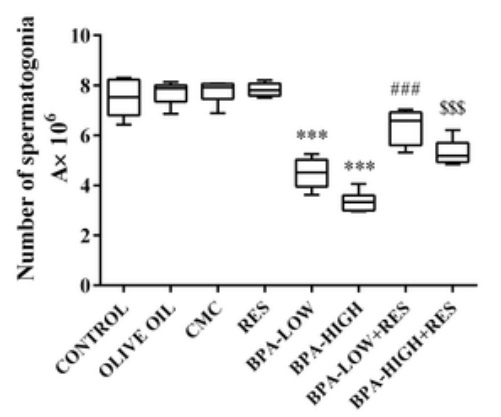

C



$\mathbf{E}$

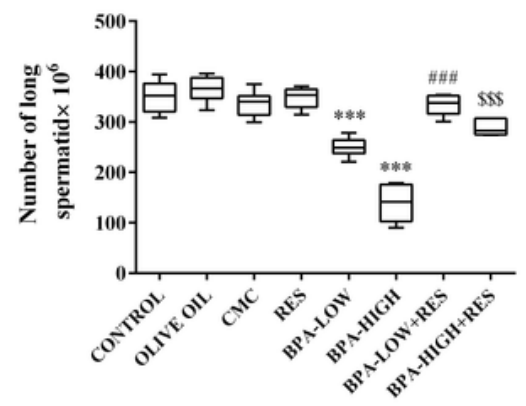

B

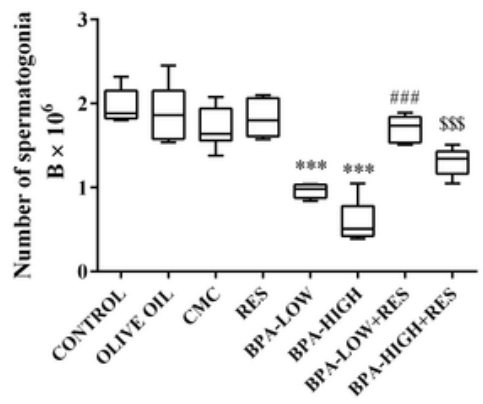

D

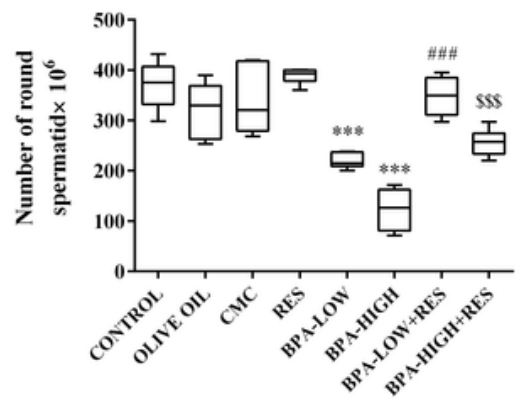

F



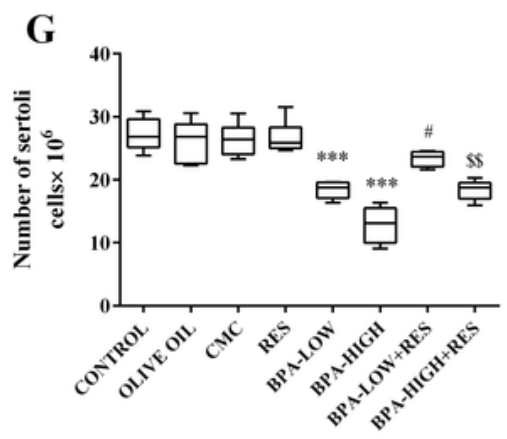

Figure 3

The box plots represents the number of spermatogonia $A(A)$, spermatogonia $B(B)$, spermatocytes $(C)$, round spermatids $(D)$, long spermatids $(E)$, Leydig $(F)$, and Sertoli $(G)$ in the control (CON), olive oil, carboxy methylcellulose (CMC), resveratrol (RES), low dose of Bisphenol A (BPA), high dose of BPA, low dose of BPA+RES, and high dose of BPA+RES groups. $n=6$ in each group. Data are presented as mean \pm SE. $* * * p<0.001$ vs. control; $\# p<0.05$, \#\#p < 0.01, and \#\#\#p < 0.001 vs. low dose of BPA; 


$$
p<0.01 \text { and }
$$

$\$ p<0.001$ vs. high dose of BPA.

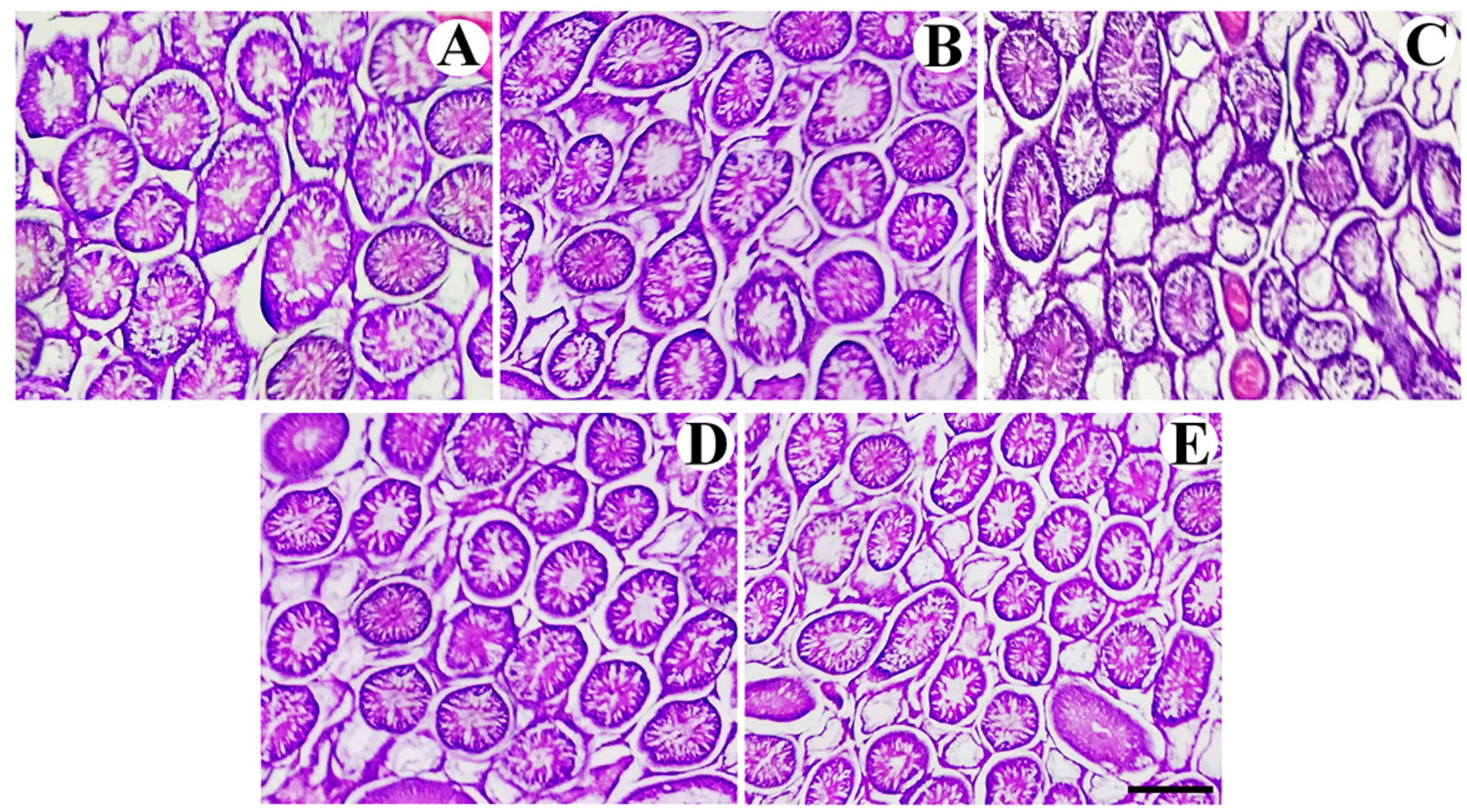

Figure 4

Representative photomicrographs of testis sections stained with hematoxylin \& eosin (H\&E) in the control $(A)$, low dose of BPA (B), high dose of BPA (C), low dose of BPA+RES (D), and high dose of BPA+RES (E) groups. Scale bar $=200 \mu \mathrm{m}$. 
A

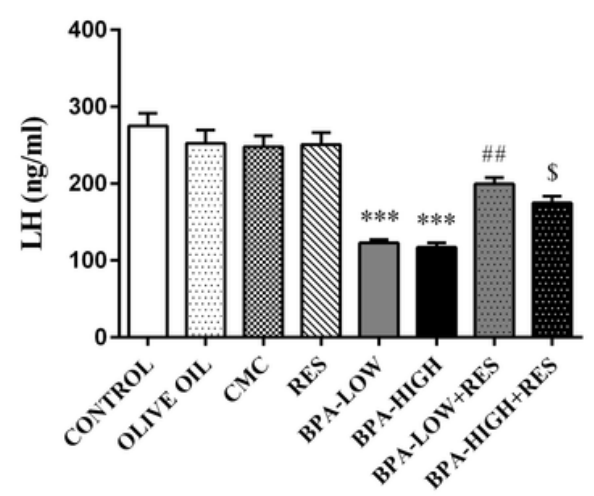

B

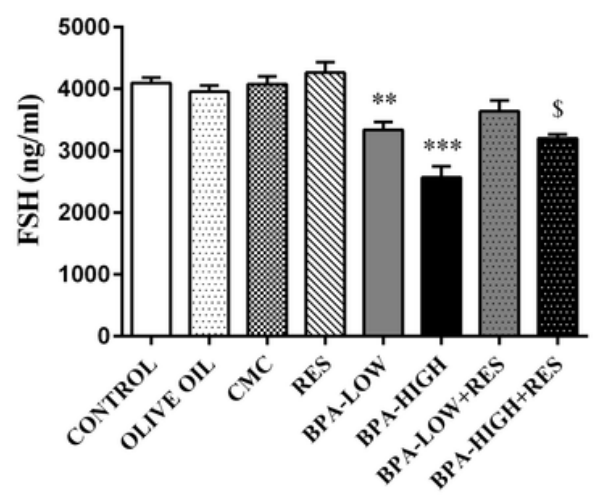

C

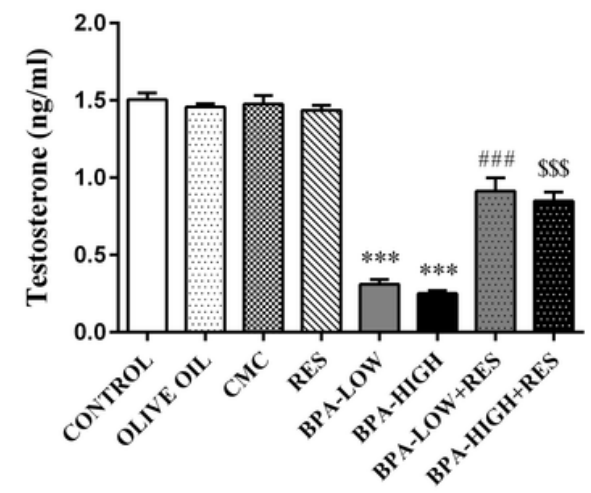

Figure 5

Serum concentrations of $\mathrm{LH}, \mathrm{FSH}$, and testosterone in the experimental groups. $\mathrm{n}=6$ in each group. Data are presented as mean \pm SE. ${ }^{* *} p<0.01$, and $* * * p<0.001$ vs. control; $\# p<0.05$, \#\#p $<0.01 v s$. low dose of BPA; $\$$ \$p $<0.01$ vs. high dose of BPA. 